\title{
Diagnosis of Bearing Outer Race Faults Using a Low-Cost Non-Contact Method with Advanced Wavelet Transforms
}

\author{
Madhusudhana Reddy Barusu ${ }^{1}$, Umamaheswari Sethurajan ${ }^{2}$, Meganathan Deivasigamani ${ }^{3}$ \\ ${ }^{1}$ Department of Electronics and Communication Engineering, \\ Veltech Hightech Dr. Rangarajan Dr. Sakunthala Engg. College, \\ Avadi, Chennai, India \\ ${ }^{2}$ Department of Information Technology, Anna University MIT Campus \\ Chrompet, Chennai, India \\ ${ }^{3}$ Department of Electronics, Anna University MIT Campus \\ Chrompet, Chennai, India \\ madhusudhanareddy@velhightech.com
}

\begin{abstract}
This study analyses the vibration of Squirrel Cage Induction Motor (SCIM) with bearing faults using highfrequency signal (Handheld UWB radar) and Software Phase Locked Loop algorithm (SPLL). The contact or non-contact methods perform condition monitoring of the SCIM. The proposed method is a non-contact technique to perform condition monitoring of the SCIM. The contact methods execute via vibration, instantaneous frequency, rotor speed and flux signals analysis; whereas non-contact methods accomplish via acoustic, current and stray flux measurement. The existing techniques suffer from the influence of adjoining electrical machines; require human expertise to mount sensors and analysing the signals. In this paper, a new, non-contact method proposed for bearing fault identification in the SCIM. The proposed method uses a high-frequency signal projected on the motor and the reflected signal captured. The signal obtained is analysed with an advanced signal processing algorithm like Rational Dilation Wavelet Transform (RDWT) to identify the faults in the SCIM. The signal energy at the fault frequency level increases from $4.72 \%$ to $5.82 \%$ with the increase in the number of the faults. The signal energy variation indicates the severity of the faults. From the experimental results, the bearing fault of the motor identified in the beginning stage of the fault.
\end{abstract}

Index Terms-Fault diagnosis; Induction motor; Phase Locked Loop algorithm; Radar signal processing; Rational dilation wavelet transform (RDWT).

\section{INTRODUCTION}

Bearings are crucial components of the IMs, which play a vital role in the operation. IMs experience the various internal faults like bearing faults, rotor bar faults, the stator winding short circuit faults and external voltage fluctuations. Among all these faults, the defects in the bearing cause approximately $51 \%$ of motors to fail. Hence, the bearing condition monitoring is crucial from industry's point of view [1]-[3]. The bearings in the IMs fail due to various factors like contamination, corrosion, misalignment, improper installation, no lubrication [4], voltage variation,

Manuscript received 21 January, 2018; accepted 16 October, 2018. overloading and unbalance supply voltage conditions.

The fluctuations in the supply voltage to IMs cause a negative sequence current flow in the rotor and the stator. The negative sequence current causes more power losses, which causes higher temperatures at bearings and other parts. The higher temperature makes surface roughness known as electrical fluting [5]. The continued operation of rough surface or surface pitting in industry causes multipoint defects in bearings like a defect in the outer race, inner race, balls and cage. The multiple faults in bearings are also caused by the air gap eccentricity in the motor, which further leads to the development of Unbalanced Magnetic Pull (UMP). UMP causes the rubbing of the rotor with the stator and the vibrations created by multiple faults in the motor is challenging to analyse, require more accurate, highly sensitive sensors for detection and analysis [6]. The parameters of the SCIM like current [7], vibration [8], temperature, acoustic emission and flux around the machine are monitored to identify the faults in the early stage. The Park's and Concordia approach [5], measures stator current, based on the circle or elliptic patterns determines the inner and outer race bearing fault. However, these methods accuracies depend on the quality of the power. The localized bearing fault diagnosis with the spectral kurtosis and envelope analysis [9], statistical measurement [10] by measuring the stator current and stray flux respectively. The flux measurement accuracy depends on the sensing coil location and distance from the motor. The wireless sensor networks are another method to identify the inner and outer race defects, which measures vibration, current and acoustic emission for fault identification [11]. The technique suffers from less accurate data delivery and data loss in the industrial environment, due to improper mounting of sensors, other machines disturbance and higher in cost due to a number of sensors. The various methods in wireless sensor network like inductive coupling principle and a triaxial accelerometer had employed in [12] to detect single point defects in the outer and inner race by using motor vibration as a medium. The amplitude modulation [13], the 
tri-axial integrated circuit with piezoelectric accelerometer and Integrated Circuit Piezoelectric (ICP) accelerometer [14] are the methods to identify the fault, by measuring motor vibration, speed, current [15], and energy loss. However, the methods suffer from the complex analysis of the data, long time for processing and continuous operation of the piezoelectric accelerometers causes overheating of sensors, which in turn require a temperature compensation circuit, so, soft-computing methods are the alternate solution for hardware methods.

The soft-computing methods have advantages like easy to implement and economical compared to hardware methods for identifying the bearing fault. The soft-computing techniques such as neural network [16], [17], pattern recognition, fuzzy logic [18], [19] have been employed to determine the bearing fault, which analyses the vibration signal of the motor. In general, the soft-computing methods, process signals by FFT, STFT and Wavelets. However, softcomputing methods suffer from computational complexity; require large computer memory, more processing time and skilled personnel in analysing the signal to identify the fault. The primary objective of this paper is to propose a low cost, a non-contact and simple method to detect, diagnose the defects in the bearings of IM by UWB radar signal analysis using an advanced signal processing technique like wavelet transforms.

The rest of the paper is organized as follows: a literature survey discussed in Section II. In Section III, bearing faults and characteristic frequencies explained. Section IV describes the details of wavelet transforms and Section V shows the framework of the proposed method. Section VI describes the experimental results and finally, Section VII concludes our work.

\section{LITERATURE SURVEY}

The bearing faults in IMs identified from acquired current and vibration signal, by extracting the pattern of the fault signal using soft computing techniques. The current signals are analysed with Artificial Neural Networks (ANNs) methods to infer the stages of the fault condition in the motor [5]. The back-propagation, feed-forward network algorithm and Park's vector algorithm are various algorithms for training the ANN. The bearing faults in the IMs such as an inner raceway, outer raceway, ball fault identify using parks algorithm and frequency signatures of the stator current with more accuracy at the early stage of fault occurrence. However, the neural network requires the same level of fault all the time.

The vibration signals are also analysed with ANNs to identify the bearing fault occurrence in the SCIMs [20]. The FFT, STFT and wavelet are various algorithms for frequency signature analysis of the acquired signal [21]. The different faults in the SCIMs caused for generation of multiple harmonics in line current. The harmonic order tracking [22], identify various faults in the early stage with the harmonics generated in the SCIMs. Whereas determining the location of fault creation in the motor is still challenging task. So, the condition monitoring of the SCIMs needs more precise, accurate fault detection and fault location with the efficient non-contact method.

The non-contact methods make use of stray flux measurement, acoustic emission measurement, vibration and voltage measurement. The stray flux around the machine is measured by a flux probe at different locations of the motor like on body and fan end. The flux probe suffers from voltage attenuation [10]. The other non-contact method is multi-sensor wireless system, which employs different sensors on the common wireless platform for measuring acoustic signal, vibration and voltage [11]. The multi-sensor method suffers from data loss while transmitting from the wireless system to base. Therefore, we propose a novel, low-cost, non-contact and simple technique for diagnosing the bearing fault in SCIMs with handheld UWB radar. The acquired signals are analysed with FFT, STFT and wavelets.

\section{BEARING FAULTS AND CHARACTERISTIC FREQUENCIES}

The SCIMs consists of two bearings at two ends of the rotor. In some motors, two bearings are the same size, whereas in others they are different sizes. The bearings used in the SCIMs comes in two versions, i.e., a sealed and a shielded version with extension $2 \mathrm{RS}$ and $\mathrm{ZZ}$ respectively. The $6205-\mathrm{ZZ}$ is a $25 \mathrm{~mm}$ ball bearing that is used in this application. The $6205-\mathrm{ZZ}$ ball bearing consists of an inner and outer raceway, with a set of rolling elements running on their tracks. The rolling element in a bearing fixed in a cage that ensures equal spacing prevents slipping from the position and mutual contacts. Figure 1 provides the geometry of the bearing NBC 6205ZZ employed in the present work and the detailed specifications of the bearing are given (Table I).

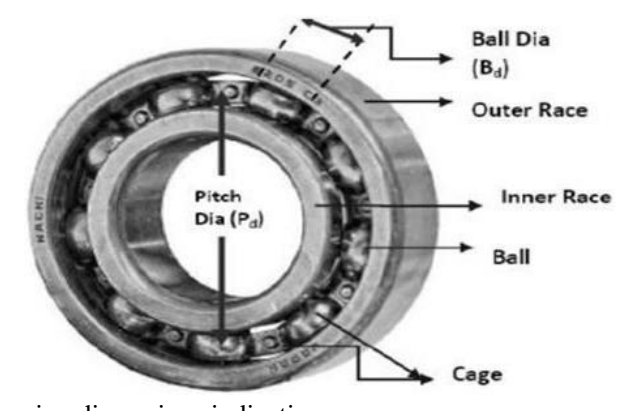

Fig. 1. Bearing dimensions indication.

\begin{tabular}{|c|c|c|}
\hline S.NO & PABLE I. 6205ZZ BEARING SPECIFICATIONS. \\
\hline 1 & MATERIAL & VALUE \\
\hline 2 & $\begin{array}{c}\text { Chrome steel } \\
\text { DIAMETERTER }\end{array}$ & $25 \mathrm{~mm} \& 52 \mathrm{~mm}$ \\
\hline 3 & BEARING WIDTH & $15 \mathrm{~mm}$ \\
\hline 4 & BALL DIA (Dbb) & $7.94 \mathrm{~mm}$ \\
\hline 5 & PITCH DIA (Dcb) & $39.4 \mathrm{~mm}$ \\
\hline 6 & NO OF BALLS (NB) & 9 \\
\hline
\end{tabular}

The dynamics of the bearings had described by the fundamental motions, which corresponds to a particular frequency [23]. These frequencies are denoted as the shaft rotational frequency $\left(F_{S R}\right)$, the fundamental cage frequency $\left(\mathrm{F}_{\mathrm{FC}}\right)$, the ball pass inner raceway frequency $\left(\mathrm{F}_{\mathrm{BPIR}}\right)$, the ball pass outer raceway frequency $\left(\mathrm{F}_{\mathrm{BPOR}}\right)$ and the ball rotational frequency $\left(\mathrm{F}_{\mathrm{RB}}\right)$. The bearings vibrate with periodical movements. So, signal processing techniques such as FFT, STFT and wavelets [24], [25] is used to extract the vibration features from frequency domain easily. 


\section{A. Shaft Rotational Frequency $\left(F_{S R}\right)$}

The bearing is part of the rotating motor system and it will rotate at the same speed $\left(F_{S R}\right)$ as the rotor or shaft speed. All other frequencies of bearings are functions of the shaft rotational frequency.

\section{B. Fundamental Cage Frequency $\left(F_{F C}\right)$}

The fundamental cage frequency developed by the motion of the cage measured as the mean of the linear velocities of the inner raceway $V_{i r}$ and the outer raceway $V_{o r}$ is described by (1-2) [18], [26] i.e.

$$
\begin{gathered}
V_{c r}=\frac{\left(V_{i r}+V_{o r}\right)}{2}, \\
F_{F C}=\frac{V_{c r}}{r_{c}}=\frac{\left(V_{i r}+V_{o r}\right)}{2 \times r_{c}}=\frac{V_{i r}+V_{o r}}{D_{c b}},
\end{gathered}
$$

where $V_{c r}$ is the linear velocities of the ball center, $D_{c b}$ is the bearing cage diameter and radius of the cage $\left(r_{c}\right)$ $=D_{c b} / 2$. The linear velocities $V_{i r}$ and $V_{\text {or }}$ represented with their respective rotational frequencies, i.e., inner rotational frequency $F_{i r}$ and outer rotational frequency $F_{o r}$ multiplied by their corresponding inner radii $r_{i}=r_{c}-\left(D_{b b} \cos \theta\right) / 2 \quad$ and outer radii $r_{o}=r_{c}+\left(D_{b b} \cos \theta\right) / 2 . D_{b b}$ is the ball diameter and $\theta$ is the contact angle of the bearing. Thus, $F_{F C}$ can be further expressed as (3) [18], [26]

$$
\begin{gathered}
F_{F C}=\frac{F_{i r} r_{i}+F_{o r} r_{o}}{D_{c b}}= \\
=\frac{1}{D_{c b}}\left(F_{i r} \frac{D_{c b}-D_{b b} \cos \theta}{2}+F_{o r} \frac{D_{c b}+D_{b b} \cos \theta}{2}\right) .
\end{gathered}
$$

\section{Ball Pass Outer Raceway Frequency $\left(F_{B P O R}\right)$}

The ball present in the bearing will pass a point at a rate on the outer raceway track known as Ball Pass Outer Raceway Frequency $\left(F_{B P O R}\right) . F_{B P O R}$ is equal to the number of bearing balls $N_{B}$ multiplied by the difference between the fundamental cage frequency $F_{F C}$ and the outer rotational frequency $F_{\text {or }}$, given by (4) [18], [26]

$$
\begin{aligned}
& F_{B P O R}=N_{B}\left|F_{F C}-F_{\text {or }}\right|= \\
& =N_{B}\left|\frac{F_{i r}\left(r_{C}-\frac{D_{b b} \cos \theta}{2}\right)+F_{o r}\left(r_{C}+\frac{D_{b b} \cos \theta}{2}\right)}{D_{c b}}-F_{o r}\right|= \\
& =\frac{N_{B}}{2}\left|\left(F_{i r}-F_{o r}\right)\left(1+\frac{D_{b b} \cos \theta}{D_{c b}}\right)\right| \text {. }
\end{aligned}
$$

\section{Ball Rotational Frequency $\left(F_{R B}\right)$}

The ball in the bearing rotates at a specific speed about its axis, which is known as the ball rotational frequency $\left(F_{R B}\right)$. This frequency can be calculated from either $F_{B P O R}$, given by (5) [18], [26]

$$
\begin{gathered}
F_{R B}=\left|\left(F_{i r}-F_{c r}\right) \frac{r_{i}}{r_{b}}\right|= \\
=\left|\left(F_{o r}-F_{c r}\right) \frac{r_{o}}{r_{b}}\right|=\frac{D_{c b}}{2 D_{b b}}\left|\left(F_{i r}-F_{o r}\right)\left(1-\frac{D_{b b}^{2} \cos ^{2} \theta}{D_{c b}^{2}}\right)\right|,
\end{gathered}
$$

where $r_{b}$ is the ball radius.

In a motor, the outer raceway of the bearing gets locked by an external casing, so, it can be assumed stationary, while the inner raceway is rotating at the speed of the shaft, i.e., $F_{o r}=0$ and $F_{i r}=F_{S R}$. Therefore, in a motor system, (3)(5) can be rewritten as (6 to 8) [18], [26]:

$$
\begin{gathered}
F_{F C}=\frac{1}{2} F_{S R}\left(1-\frac{D_{b b} \cos \theta}{D_{c b}}\right), \\
F_{B P O R}=\frac{N_{B}}{2} F_{S R}\left(1-\frac{D_{b b} \cos \theta}{D_{c b}}\right), \\
F_{R B}=\frac{D_{c b}}{2 D_{b b}} F_{S R}\left(1-\frac{D_{b b}^{2} \cos ^{2} \theta}{D_{c b}^{2}}\right) .
\end{gathered}
$$

The bearings in induction motor with defects will produce vibrations with any one of the frequencies among above described three frequencies.

\section{RATIONAL-DILATION WAVELET TRANSFORMS}

The SCIMs develop a vibration of the specific frequency for different faults. The fault frequency identified with signal analysis algorithms like FFT, STFT and wavelet transforms. These algorithms are described below.

\section{A. FFT}

All the signals are classified as stationary signals and nonstationary signals. The stationary signals are analysed by Fourier analysis. The FFT analysis of any signal $y[n]$ is calculating Discrete Fourier Transform (DFT) with reduced computational complexity. $N$ is the number of $x[n]$ samples. The FFT of the signal is given by (9) [27]

$$
Y(\omega)=\sum_{n=0}^{N-1} y[n] e^{-j \omega n T},
$$

where $Y(\omega)$ is DFT of $y[n]$.

\section{B. STFT}

The FFT analysis is suitable for stationary signals, whereas not appropriate to analyse a non-stationary signal. The non-stationary signals have transitory characteristics such as drifts, abrupt changes, and frequency trends [1]. So, the non-stationary signals can be analysed by considering the small portions of the signals. The technique of analysing small portions of the signal is known as Short Time Fourier Transform (STFT). The STFT of an input signal $x(t)$ with frequency $t$ is given by (10) [28]

$$
\operatorname{STFT}_{X}^{(\omega)}\left(t^{\prime}, f\right)=\int_{t}\left[x(t) \times \omega *\left(t-t^{\prime}\right)\right] \times e^{-j 2 \pi f t} d t
$$


where $\omega^{*}\left(t-t^{\prime}\right)-$ window function centered at time $t=t^{\prime}$.

\section{Rational-Dilation Wavelet Transform}

The STFT of the signal provides limited precision and that precision is determined by the size of the window. The fixed size of the window is the main drawback of the STFT [29]. The wavelet transform overcomes the difficulties mentioned above. A windowing technique with the variablesize region is then used to perform the signal analysis. Wavelet analysis allows the use of long time intervals for more precise low-frequency information and shorter regions for high-frequency details [30]. The discrete wavelet transform (DWT) consists of sampling the scaling and shifted parameters, but not the signal or the transform; this leads to high-frequency resolution at low frequencies and high-time resolution at higher frequencies.

A discrete signal $x[n]$ could be decomposed as given in (11) [28], where $\varphi[n]$ is the scaling function, $\phi[n]$ is the mother wavelet, $\varphi_{i o, l}[n]=2^{i o / 2} \varphi\left(2^{i o} n-l\right)$ is the scaling function at a scale of $s=2^{i o}$ shifted by $l$, $\phi_{i, l}[n]=2^{i / 2} \phi\left(2^{i} n-l\right)$ is a mother wavelet at a scale of $s=2^{i}$ shifted by $l, a_{i o, l}$ is the approximation coefficient at a scale of $s=2^{i o}$ and $d_{i, l}$ is the detail coefficient at a scale of $s=2^{i o}$

$$
x[n]=\sum_{l} a_{i o, l} \varphi_{i o, l}[n]+\sum_{i=i o}^{I-1} \sum_{l} d_{i, l} \phi_{i, l}[n] .
$$

In this paper, SCIMs bearing condition is monitored by analysing the vibration signal, so, a suitable wavelet transform needs to be chosen. There is a number of wavelet transforms available for vibration signal analysis like cosine modulated filter banks, wavelet packets and dyadic wavelet [31]. The dyadic wavelet transform is an easily invertible 'constant- Q' transform that is very effective for the sparse representation of piecewise smooth signals [32]. However, the dyadic WT is less efficient for processing vibration measurements. Vibration signals are quasi-periodic over short-time intervals and for analysing those signals needs better frequency resolution than that provided by the dyadic WT. The dyadic WT has a very low Q-factor and poor frequency resolution. Other transforms, like the cosine modulated filter banks and wavelet packets, are often used for vibration signals instead of the dyadic WT. But these transforms do not have constant-Q. So, in this paper, a wavelet transform having constant-Q is used .i.e. RDWT. RDWT can be implemented as critically sampled and overcomplete RDWT. The overcomplete RDWT provides several advantages compared to the critically sampled RDWT, such as minimal-length perfect reconstruction filters, shift-invariant and smooth analysis/synthesis functions [33].

\section{FRAMEWORK}

The framework of the proposed non-contact method for the bearing fault identification in SCIMs is shown in Fig. 2. In the proposed method, a high-frequency signal from the handheld UWB radar is projected on the motor and the reflected signal is captured without any contact with SCIM. The acquired signal is fed to SPLL. The signal given to SPLL is compared with the feedback signal, from which error signal is obtained. The error signal received is processed with the various signal processing algorithms and the fault is identified.

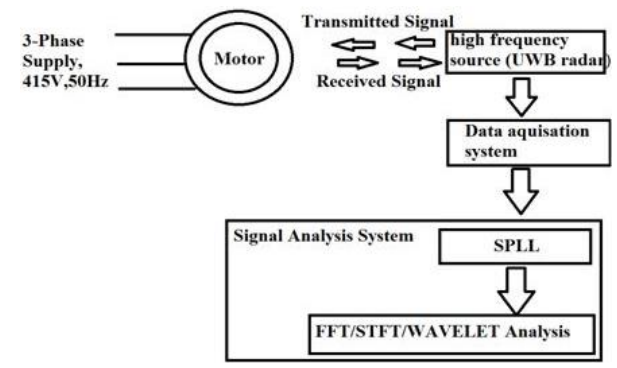

Fig. 2. The framework of the proposed system.

In the proposed method, the main components are SCIM, high-frequency signal source (UWB radar) and data acquisition system with SPLL algorithm.

\section{A. Induction Motor and UWB Radar}

The device under test is a $2 \mathrm{Hp}$ three-phase SCIM. The critical component of the proposed system is UWB radar (high-frequency signal source). Radar works on the fundamental principle of transmitting electrical pulse signal towards the target and receiving the reflected signal. The receiver processes the reflected signal for various parameters of the target like speed, harmonics and direction of motion. UWB radar acts as the high-frequency signal source in the proposed system, which is projected onto the motor and the signal reflected from the motor is captured.

The radar system contains three different sub-blocks, i.e., transmitter, receiver and antenna system [34]. In the proposed technique, an $\mathrm{HB}$ series of microwave sensor module is used (Fig. 3). The sensor is of X-Band Monostatic Dielectric Resonator Oscillator (DRO) Doppler transceiver front-end module. The radar module works with 5V DC supply and generates a high-frequency signal (10.525 GHz frequency). The module consists of DRO, microwave mixer and patch antenna. Furthermore, the patch antenna transmits high-frequency signal on to the target via a transmitter (TX). The receiver (RX) antenna receive reflected signal and fed to the mixer. The mixer mixes the transmitted and received signals and generate a signal with a frequency equal to the difference of those two.

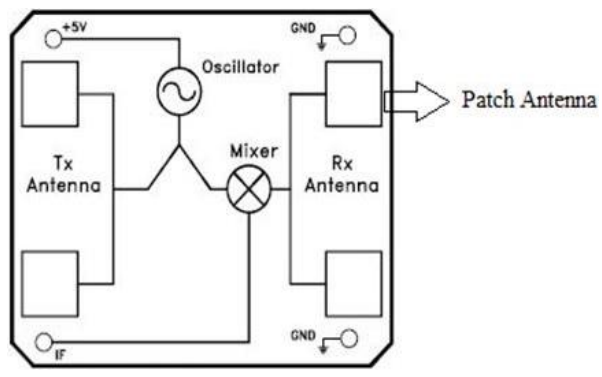

Fig. 3. UWB radar module block diagram.

The proposed method works based on the international 
patent application. According to the patent application [35], "a microwave signal can be amplitude modulated by a mechanically oscillating object in the signal path between a transmitter and receiver." Therefore, transmitted microwave signal modulated according to the characteristic frequency of SCIM vibration.

The handheld UWB radar emits a high-frequency signal $x\left(t_{1}\right)$ (sinusoidal signal) with a frequency $f_{1}$ and amplitude $M_{1}$ represented as (12)

$$
x\left(t_{1}\right)=M_{1} \cos \left(2 \pi f_{1} t_{1}\right) .
$$

The SCIM vibrates with a characteristic frequency $f_{2}$ and amplitude $N_{1}$. The vibration signal $y\left(t_{1}\right)$ is represented as (13)

$$
y\left(t_{1}\right)=N_{1} \cos \left(2 \pi f_{2} t_{2}\right) .
$$

The $x\left(t_{1}\right)$ is amplitude modulated with $y\left(t_{1}\right)$ and the resultant signal $z\left(t_{1}\right)$ is (14)

$$
z\left(t_{1}\right)=\left\lfloor 1+y\left(t_{1}\right)\right\rfloor \times x\left(t_{1}\right)=x\left(t_{1}\right)+x\left(t_{1}\right) \times y\left(t_{1}\right) .
$$

Based on mathematical identities [36], [37], $z\left(t_{1}\right)$ shown as, (15)

$$
\begin{gathered}
z\left(t_{1}\right)=M_{1} \cos \left(2 \pi f_{1} t_{1}\right)+\frac{M_{1} N_{1}}{2} \times \\
\times\left[\cos 2 \pi\left(f_{1}+f_{2}\right) t_{1}+\cos 2 \pi\left(f_{1}-f_{2}\right) t_{1}\right] .
\end{gathered}
$$

Therefore, the resultant signal can be written as the sum of the three sine waves, i.e., the high-frequency signal $\left(x\left(t_{1}\right)\right)$ and two sideband sine waves with sum and difference of two signal frequencies. Furthermore, the acquired UWB radar signal analysed with FFT and frequency components obtained. The obtained frequency components indicate the fault condition in SCIM.

\section{B. Data Acquisition System with SPLL}

The other component of the proposed method is a data acquisition system with SPLL. The signals captured by UWB radar are given to a data acquisition system (SIGVIEW software). The acquisition system records the signal and the recorded signal is input to the SPLL for obtaining the error signal for analysing the faults in the SCIM. SPLL is an algorithm, where all the functions of the PLL are realized by the program. SPLL is more advantageous compared with hardware PLL as SPLL work faster, immune to ambient conditions, more accurate and is reconfigurable [38]. The SPLL gives the output error signal, which is analysed with various signal processing algorithms.

Algorithm of Software Phase Locked Loop (SPLL)

1. Set sampling frequency, supply frequency, time of simulation

2. Provide input reference signal obtained from the faulty motor to phase detector.

3. Provide output of phase detector to the loop filter.

4. Given output of the filter to Digitally Controlled Oscillator (DCO)

$\&$ calculate sine and cosine using integration process

5. Feedback the DCO output to the phase detector and obtain error signal from phase detector to analyse the fault in IM.

\section{Results AND DisCUSSION}

The parameters of 3-Phase SCIMs for conducting experiments are tabulated (Table II).

TABLE II. THREE PHASE SCIMS PARAMETERS.

\begin{tabular}{|c|c|c|}
\hline S. No. & PARAMETER & RATED VALUE \\
\hline 1 & Power & 2HP $(1.5 \mathrm{KW})$ \\
\hline 2 & Current & $3.5 \mathrm{~A}$ \\
\hline 3 & Synchronous speed & $1500 \mathrm{rpm}$ \\
\hline 4 & Speed & $1410 \mathrm{rpm}$ \\
\hline 5 & Supply voltage & 3 -Phase, $415 \mathrm{~V}, 50 \mathrm{~Hz}$ \\
\hline
\end{tabular}

Figure 4(a) and Fig. 4(b) shows the experimental setup. The experimental setup consists of

1. Three-phase Squirrel Cage 2-HP IM with brake drum loading setup;

2. Voltmeter, Ammeter and tachometer for measuring voltage, current and speed;

3. UWB radar for generating and receiving signal;

4. PC equipped with data acquisition software SIGVIEW and SPLL.

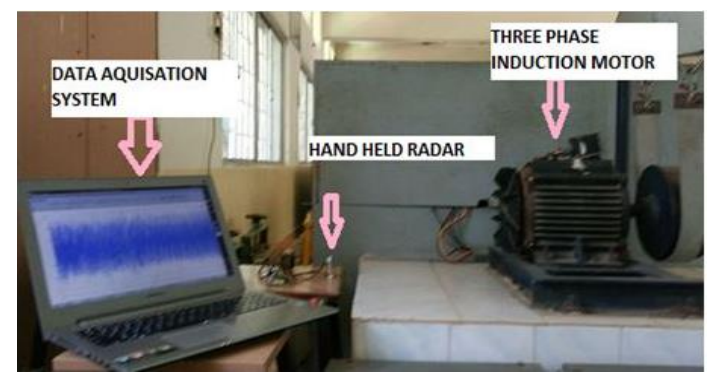

(a)

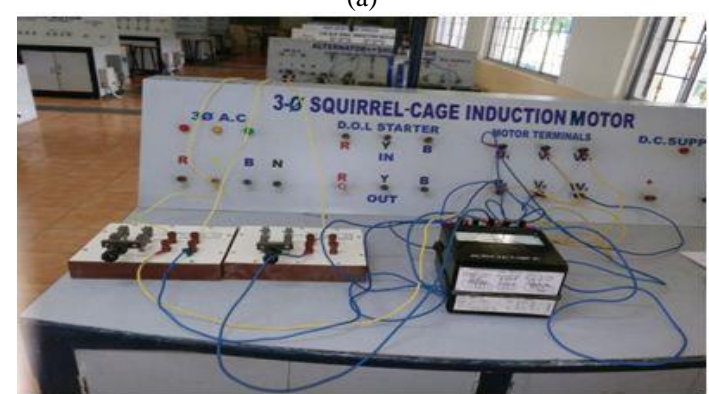

(b)

Fig. 4. Experimental setup for bearing fault identification.

The UWB radar is arranged at a fixed distance from the motor and the reflected signal received by the radar is recorded using data acquisition software (SIGVIEW) with a sampling rate of $10000 \mathrm{~Hz}$ (sampling period of $0.1 \mathrm{~ms}$ ). The first $2.5 \mathrm{~s}$ of signal information is processed for signal analysis. The signal is analysed and plotted on a graph. The various faults occur in bearings of the SCIM are identified through radar reflected signal with an experimental setup.

In the experimental setup, the various bearing faults are created and the corresponding signals are recorded. The bearing faults are made artificially by super drilling the hardened material bearing. Figure 5 shows the different bearing faults. Based on the parameters of bearing listed in the table and considering that the motor is operating at the measured shaft speed of $1498 \mathrm{rpm}(\mathrm{Fs}=24.96 \mathrm{~Hz})$, the characteristic vibration frequencies are calculated from (6)(8) as listed below [20] (Table III). 
TABLE III. CHARACTERISTIC DEFECT FREQUENCY

\begin{tabular}{|c|c|c|}
\hline Sl. No. & Fault Location & Characteristic defect frequency \\
\hline 1 & Normal & $24.96 \mathrm{~Hz}$ \\
\hline 2 & Outer race & $89.18 \mathrm{~Hz}$ \\
\hline 3 & Balls & $116 \mathrm{~Hz}$ \\
\hline
\end{tabular}

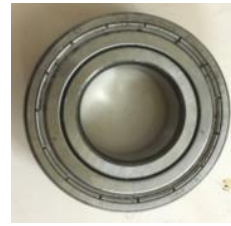

(a)

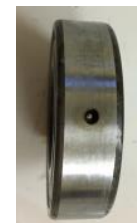

(b)

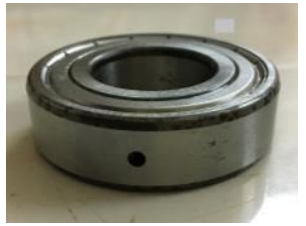

(c)
Fig. 5. Rotor bearings in three-phase induction motor with various faults (a) normal, (b) outer race fault, (c) outer race and ball fault.

The error signal from SPLL is analysed with FFT, STFT and RDWTs. The outputs of these signal analysis algorithms are explained below.

\section{A. FFT Analysis}

The 3-phase SCIMs operate with 3-phase, $415 \mathrm{~V}, 50 \mathrm{~Hz}$ supply and full load current of $3.5 \mathrm{~A}$.

In the proposed method, the bearing faults in the 3-phase SCIM are identified with the non-contact method. The SCIM consists of two bearings, one towards load end and another at the opposite end of the rotor. In the two bearings of the SCIM, one bearing is damaged artificially and tried to identify the faults with the proposed method. There are two bearings under test, which have one fault (outer race fault) and two faults (outer race and ball fault). The faults are created by drilling a hole of $3 \mathrm{~mm}$ with a special drill. Figure 6 shows the power spectra of the SCIM operating with multiple fault bearing. The signal is processed with proposed method and analysed with FFT. The power spectra maximum is $105.7 \mathrm{~dB}$ at $50 \mathrm{~Hz}$.

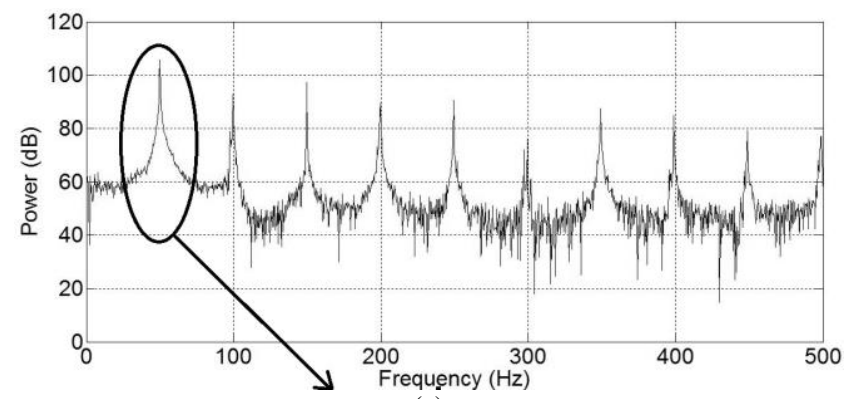

(a)

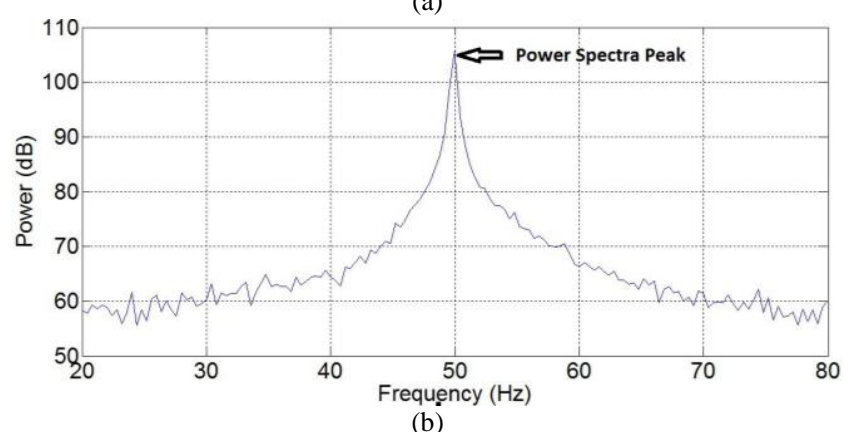

Fig. 6. Power spectra of error signal with Two Faults.

\section{B. STFT Analysis}

The power spectra of error signal with FFT analysis indicate peak value clearly, whereas the rotor harmonics are not able to distinguish. FFT analysis is suitable for external electrical fault identification, but internal fault identification is challenging "to be published" [39]. The FFT frequency resolution is not enough to show rotor harmonics. However, the FFT analysis is suitable to identify the electrical faults like voltage unbalance, load variations and supply frequency variation. So, the error signal is analysed with STFT, which is having a better frequency resolution to identify the bearing faults. The output of the signal analysis is drawn as a graph with frequency, time and the power of the signal on three axis of the graph. Figure 7 shows the power spectra of the SCIM with STFT analysis. The graph (Fig. 7(b)) is showing the rotor asymmetry harmonics at $44.2 \mathrm{~Hz}$ and $55.2 \mathrm{~Hz}$ with the more resolution than in FFT analysis.

\section{Wavelet Analysis}

The power spectra of error signal with STFT analysis indicates strong harmonics like rotor harmonics able identify, but bearing fault harmonics (outer race and ball fault harmonics) with minimum power are not observable in the STFT analysis (Fig. 7(c)). So, the error signal is analysed with wavelet transforms. Figure 8(a) to Fig. 8(c) shows the RDWT outputs for various fault conditions of the SCIM like normal, single fault (outer race fault) and two faults (outer race \& ball fault). The plot consists of waveforms of 12 levels of WTs along with actual signal recorded and the percentage of the signal power at different levels is specified. Each level of the subband carries information about a particular band frequency as is given (Table IV).

TABLE IV. FREQUENCY BANDS IN EACH LEVEL.

\begin{tabular}{|c|c|c|}
\hline \multirow{2}{*}{ LEVEL } & \multicolumn{2}{|c|}{ FREQUENCY RANGE (Hz) } \\
\cline { 2 - 3 } & FROM & TO \\
\hline L1 & 5000 & 10000 \\
\hline L2 & 2500 & 5000 \\
\hline L3 & 1250 & 2500 \\
\hline L4 & 625 & 1250 \\
\hline L5 & 312.5 & 625 \\
\hline L6 & 156.25 & 312.5 \\
\hline L7 & 78.125 & 156.25 \\
\hline L8 & 39.062 & 78.125 \\
\hline L9 & 19.531 & 39.062 \\
\hline L10 & 9.766 & 19.531 \\
\hline L11 & 4.883 & 9.766 \\
\hline L12 & 0 & 4.883 \\
\hline
\end{tabular}

In the wavelet plot, the sum of the total power is $100 \%$. Percentage of each sub band signal power is measured as the ratio of the particular level signal power to the total power. The RDWT satisfy the Parseval property, i.e., the distribution of the power across the subbands reflects the frequency content of the signal [31], [33]. So, the percentage power of each subband gives the percentage of frequency content in the total signal. The outer race fault and ball fault harmonics $(89.18 \mathrm{~Hz}$ and $116 \mathrm{~Hz})$ presence identify with the measuring the power at the level-7. The subband power analysed with MATLAB-2017 version and tabulated Table V).

TABLE V. SIGNAL POWER PERCENTAGE FOR DIFFERENT FAULT CONDITIONS WITH PROPOSED METHOD ANALYSIS.

\begin{tabular}{|c|c|}
\hline No of Faults/Level & Level-7 signal power \\
\hline Normal & $4.72 \%$ \\
\hline Single Fault & $4.82 \%$ \\
\hline Double Fault & $5.82 \%$ \\
\hline
\end{tabular}


The level-7 signal power is increasing with a number of faults $(4.72 \%$ to $5.82 \%)$. During no-fault condition, the signal power is only $4.72 \%$, with outer race fault alone, the signal power increases by $0.1 \%(4.82 \%)$ indicates that the fault frequency $(89.18 \mathrm{~Hz})$ is affecting. When bearing damage both in the outer race and ball, the signal power increases by $1.1 \%(5.82 \%)$ from the normal condition, indicates that the fault frequencies $(89.18 \mathrm{~Hz}$ and $116 \mathrm{~Hz})$, which are in the range of level-7 are dominant and affecting IM more severely.

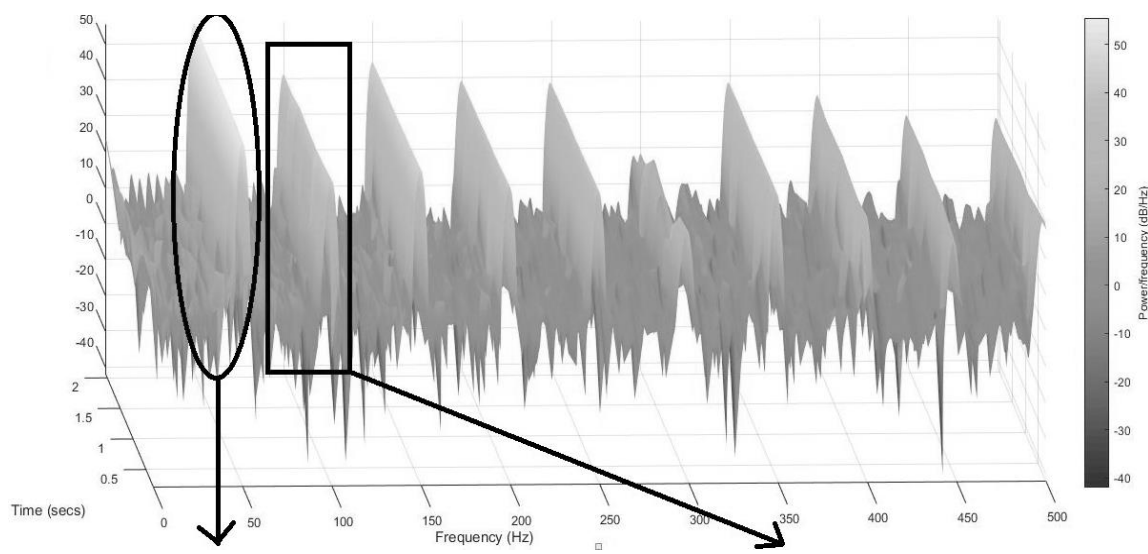

(a)

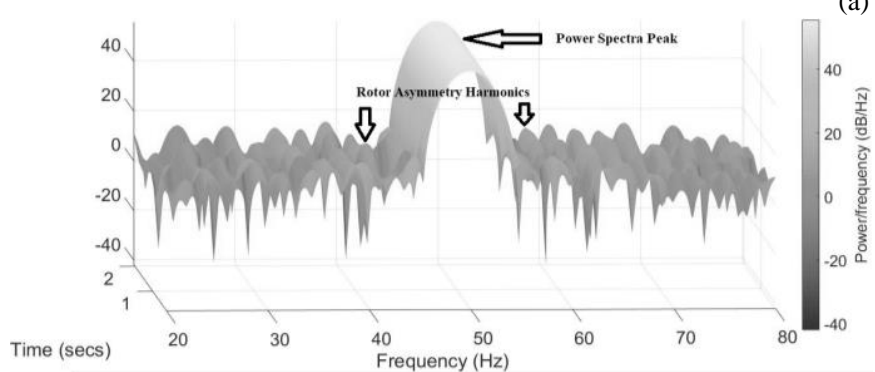

(b)

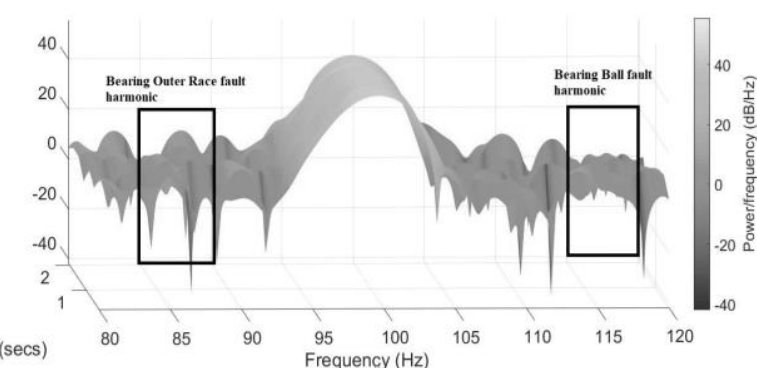

(c)

Fig. 7. Power spectra of the error signal for Two Faults with STFT analysis.

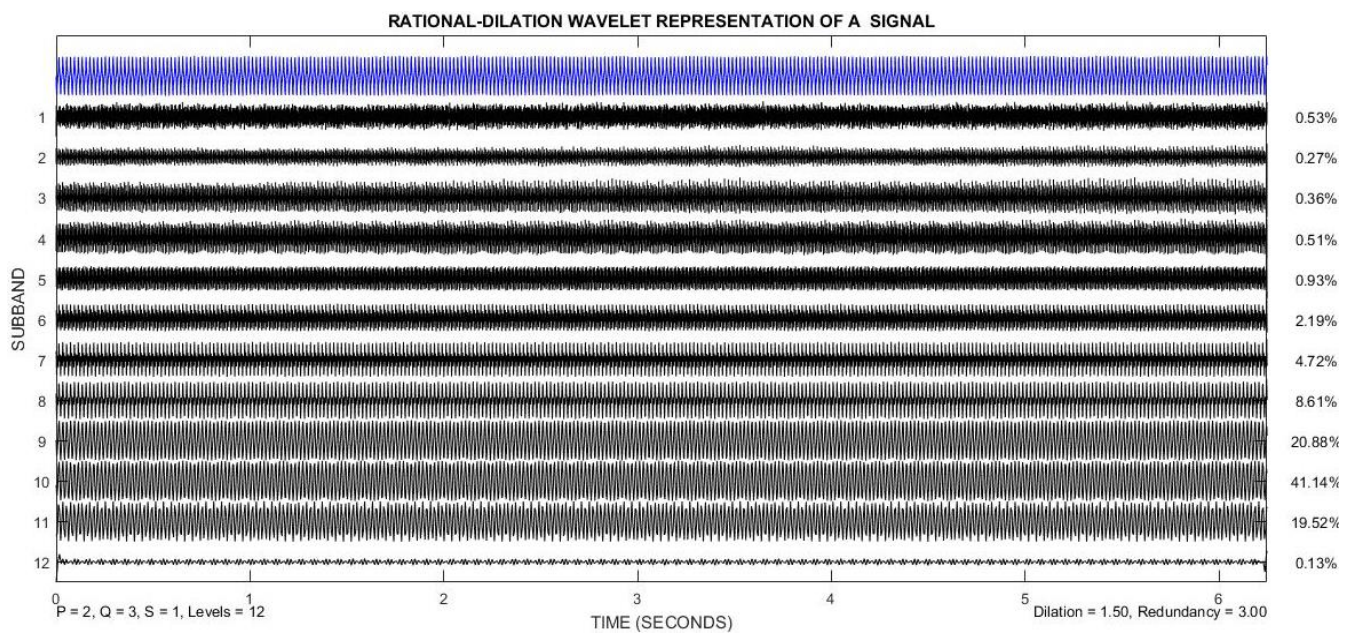

(a)

RATIONAL-DILATION WAVELET REPRESENTATION OF A SIGNAL

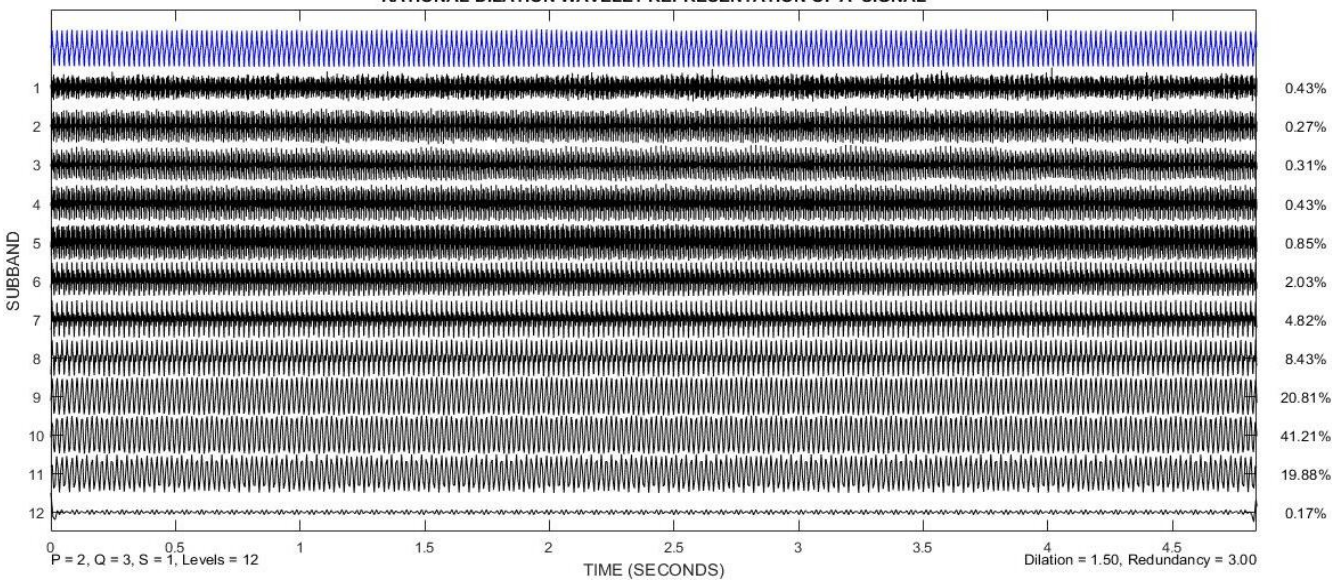

(b) 


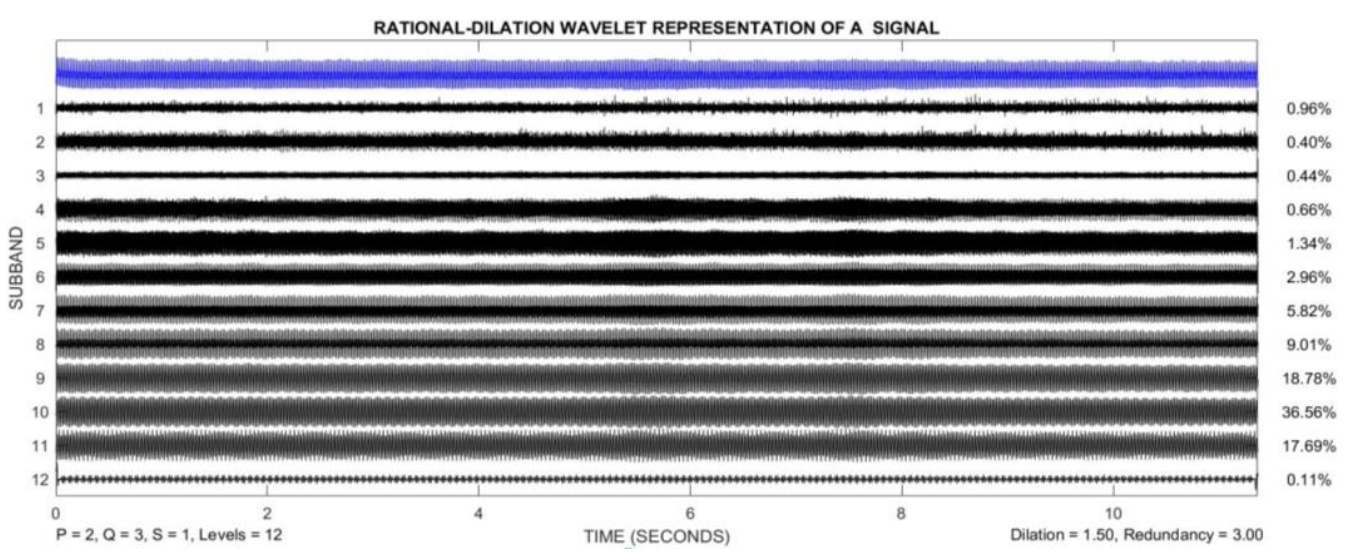

(c)

Fig. 8. Wavelet output under (a) normal condition, (b) single fault, (outer race fault), (c) two fault (outer race \& ball fault) condition.

\section{COMPARISON WITH RELATED WORK}

The below table gives the comparison for various contact and non-contact methods with the proposed method, used for bearing fault identification in the three-phase SCIM regarding signals measured, analysis technique, unique components required, signal information required and cost of the system. Multi-sensor wireless system [11] needs various sensors and sensor network (500 \$ and above) and suffers from data loss while transmitting. The temperature measurement, vibration measurement [12] method require special wireless sensor network and specially designed lab for obtaining accurate signal information, results in increasing the cost (above 1000 \$) of the experimental setup for fault identification. The stray flux measurement [10] is a non-contact method, whose price includes a unique stray flux measuring coil arrangement and data acquisition board (above 100 \$). The stray flux measurement influenced by adjoining machines flux and total flux is not possible to capture. The cost of contact methods increases due to continuous maintenance and replacement of sensors. Even though the sensors are low cost $(1 \$$ to $4 \$)$, continuous replacement increases the overall cost of the system. The other expensive experimental system required is a particular signal capturing and storage devices like DSO (above 100 \$). The Kurtosis and Envelope Analysis and rotor speed based bearing fault diagnosis methods are costly and also not able to distinguish multiple faults in bearing. When compared with other techniques, the proposed method is more economical for implementation as it does not require any costly and advanced data acquisition boards, signal storage devices, expensive sensors which need replacing regularly and special software for signal analysis. The proposed methods able identify the multiple faults in the bearing. So, the proposed method is implementable with low cost.

TABLE VI. COMPARISON OF EXISTING METHODS WITH PROPOSED METHOD.

\begin{tabular}{|c|c|c|c|c|c|c|c|c|}
\hline S. No. & $\begin{array}{l}\text { Type of } \\
\text { the } \\
\text { Method }\end{array}$ & Technique & $\begin{array}{c}\text { Signals } \\
\text { Measured }\end{array}$ & $\begin{array}{c}\text { Signal } \\
\text { Analysis } \\
\text { Technique }\end{array}$ & $\begin{array}{l}\text { Complexity (Special } \\
\text { Components } \\
\text { Required) }\end{array}$ & $\begin{array}{c}\text { Signal } \\
\text { information } \\
\text { Required }\end{array}$ & Cost & Remarks \\
\hline 1 & \multirow{3}{*}{$\begin{array}{l}\text { Non- } \\
\text { Contact } \\
\text { Method }\end{array}$} & $\begin{array}{l}\text { Proposed Method } \\
\text { (SPLL based } \\
\text { Technique) }\end{array}$ & $\begin{array}{l}\text { Vibration } \\
\text { Signal }\end{array}$ & RDWT & $\begin{array}{l}\text { Low(UWB radar, } \\
\text { SPLL Algorithm) }\end{array}$ & $2.5 \mathrm{Sec}$ & $\begin{array}{l}\text { Low (50\$ } \\
\text { and above) }\end{array}$ & $\begin{array}{l}\text { It is not affected by } \\
\text { surrounding noise } \\
\text { or temperature }\end{array}$ \\
\hline 2 & & $\begin{array}{c}\text { Means of Statistical } \\
\text { Processing } \\
\text { Technique[10] }\end{array}$ & Stray Flux & FFT & $\begin{array}{l}\text { Medium (Flux Sensor- } \\
\text { M-343F-1204, NI USB } \\
\text { 6212-acquisition } \\
\text { board) } \\
\end{array}$ & $50 \mathrm{Sec}$ & $\begin{array}{l}\text { Medium } \\
\text { (100\$ and } \\
\text { above })\end{array}$ & $\begin{array}{c}\text { The sensor coil may } \\
\text { be influenced by } \\
\text { adjoining electrical } \\
\text { machines. }\end{array}$ \\
\hline 3 & & $\begin{array}{c}\text { Multisensor } \\
\text { Wireless System } \\
\text { [11] }\end{array}$ & $\begin{array}{l}\text { Vibration, } \\
\text { Current and } \\
\text { Acoustic }\end{array}$ & HHT, FFT & $\begin{array}{c}\text { High (two-axis } \\
\text { accelerometer, Hall- } \\
\text { effect sensor, wireless } \\
\text { sensor network } \\
\text { (Imote2)) }\end{array}$ & $4 \mathrm{Sec}$ & $\begin{array}{l}\text { High (500\$ } \\
\text { and above) }\end{array}$ & $\begin{array}{l}\text { Wireless system } \\
\text { suffers from data } \\
\text { loss while } \\
\text { transmitting. }\end{array}$ \\
\hline 4 & \multirow{4}{*}{$\begin{array}{l}\text { Contact } \\
\text { Method }\end{array}$} & $\begin{array}{l}\text { Spectral Kurtosis } \\
\text { and Envelope } \\
\text { Analysis [9] }\end{array}$ & Stator Current & FFT & $\begin{array}{c}\text { Medium (Current } \\
\text { probes, monoaxial } \\
\text { accelerometer) }\end{array}$ & N/A & $\begin{array}{l}\text { Medium } \\
(100 \$ \text { and } \\
\text { above })\end{array}$ & $\begin{array}{c}\text { Kurtosis and } \\
\text { Envelope Analysis } \\
\text { can detect only } \\
\text { outer race fault. }\end{array}$ \\
\hline 5 & & $\begin{array}{c}\text { Motor Current } \\
\text { Signature Analysis } \\
\text { (MCSA) [28],[40] }\end{array}$ & Current & DWT & $\begin{array}{l}\text { Medium (data- } \\
\text { acquisition system (NI } \\
\text { MY DAQ), the current } \\
\text { sensor LEM LA55P) }\end{array}$ & N/A & $\begin{array}{l}\text { Medium } \\
\text { (100\$ and } \\
\text { above) }\end{array}$ & $\begin{array}{c}\text { Require large } \\
\text { memory to store } \\
\text { data and affected } \\
\text { with surrounding } \\
\text { Noise } \\
\end{array}$ \\
\hline 6 & & $\begin{array}{l}\text { Rotor Speed Based } \\
\text { Bearing Fault } \\
\text { Diagnosis[15] }\end{array}$ & $\begin{array}{l}\text { Vibration, } \\
\text { Current \& } \\
\text { Speed }\end{array}$ & AVB-PCA & $\begin{array}{l}\text { High ((NI)cDAQ-9178 } \\
\text { USB bus chassis, } \\
\text { NI9411 module, E60H } \\
\text { NPN type encoder) }\end{array}$ & N/A & $\begin{array}{l}\text { High (500\$ } \\
\text { and above) }\end{array}$ & $\begin{array}{l}\text { Not able to } \\
\text { distinguish the fault } \\
\text { from other sources } \\
\text { of speed oscillation }\end{array}$ \\
\hline 7 & & $\begin{array}{l}\text { Thermal-induced } \\
\text { Shift[12] }\end{array}$ & $\begin{array}{c}\text { Temperature \& } \\
\text { Vibration }\end{array}$ & FFT & $\begin{array}{l}\text { Medium(tri-axial } \\
\text { accelerometer, U2J } \\
\text { Murata capacitor, } \\
\text { Spectrum analyser) }\end{array}$ & $\begin{array}{l}\text { More than } 60 \\
\text { Sec }\end{array}$ & $\begin{array}{l}\text { High } \\
\text { (1000\$ and } \\
\text { above) }\end{array}$ & $\begin{array}{c}\text { Require unique } \\
\text { wireless sensor } \\
\text { network and lab, } \\
\text { which is more } \\
\text { costly }\end{array}$ \\
\hline
\end{tabular}


The other parameters compared are the signals acquired and analysis method for identifying the bearing faults. In the proposed method vibration signal alone analysed, whereas, in other techniques, more than one signal (vibration, current, speed and acoustic signal) is required for fault identification. The signal analysis algorithms used in the other methods are FFT [9], [12], STFT [41], Hilbert-Huang Transform (HHT) [11], DWT [28], [40] and absolute value-based principal component analysis (AVB-PCA) [15], which suffer from unstable Q-factor, whereas, in the proposed method RDWT (constant Q-factor) is utilized for signal analysis.

The complexity of experimental setup or devices utilized in the proposed method is simple as compared with other methods. The existing contact methods require a set of sensors attaching to the induction motor to collect the data and the sensor network connections increase the complexity of the system. Whereas the other non-contact methods require specialized equipment or the environment, for recording signal without any interference from the surroundings.

From all the above comparison, the proposed method is preferable as it is low cost and low complexity of experimental setup. The proposed method also had other advantages like tolerant to temperature change, humidity, and immune to the acoustic emission of other machines and not affected due to overheating of the motors during the fault condition.

\section{CONCLUSIONS}

In this paper, UWB radar-based non-contact, low-cost method proposed and implemented successfully to identify the various bearing faults. The signals obtained from UWB radar are analysed with SPLL algorithm and RDWTs. The faulty signal of first $2.5 \mathrm{~s}$ is analysed. By comparing the signal power at fault frequency levels, the faults in the bearing are identified. The signal power varies from $4.72 \%$ to $5.82 \%$ at level-7 of wavelet output, as the motor condition varies from no-fault condition to two bearing faults. It indicates with the increase of the number of faults, the fault frequency harmonic strength increasing. So, by observing the harmonics strength, fault identified. By performing many experiments, it is verified that the proposed non-contact method identifies bearing faults in the early stage and cost-effective.

\section{ACKNOWLEDGMENT}

The authors would like to thank Dr. N. R. Shanker for having helped in hardware setup and conducting experiments.

\section{REFERENCES}

[1] M. E. H. Benbouzid, "A review of induction motors signature analysis as a medium for faults detection", IEEE Trans. Ind. Electron., vol. 47, no. 5, pp. 984-993, 2000. DOI: 10.1109/41.873206.

[2] A. H. Bonnett, G. C. Soukup, "Cause and analysis of stator and rotor failures in three-phase squirrel-cage induction motors", IEEE Trans. Ind. Appl., vol. 28, no. 4, pp. 921-937, 1992. DOI: 10.1109/28.148460.

[3] P. Zhang, Y. Du, T. G. Habetler, B. Lu, "A survey of condition monitoring and protection methods for medium-voltage induction motors", IEEE Trans. Ind. Appl., vol. 47, no. 1, pp. 34-46, 2011. DOI: 10.1109/TIA.2010.2090839.

[4] S. J. Lacey, "An overview of bearing vibration analysis", Maint. Asset Manag., vol. 23, no. 6, pp. 32-42, 2008.
[5] I. Y. Onel, M. E. H. Benbouzid, "Induction motor bearing failure detection and diagnosis: park and concordia transform approaches comparative study", IEEE/ASME Trans. Mechatronics, vol. 13, no. 2, pp. 257-262, 2008. DOI: 10.1109/TMECH.2008.918535.

[6] N. Tandon, G. S. Yadava, K. M. Ramakrishna, "A comparison of some condition monitoring techniques for the detection of the defect in induction motor ball bearings", Mech. Syst. Signal Process., vol. 21, no. 1, pp. 244-256, 2007. DOI: 10.1016/j.ymssp.2005.08.005.

[7] K. C. Deekshit Kompella, M. Venu Gopala Rao, R. Srinivasa Rao, "Bearing fault detection in a 3 phase induction motor using stator current frequency spectral subtraction with various wavelet decomposition techniques", Ain Shams Eng. J., 2017. DOI: 10.1016/j.asej.2017.06.002.

[8] J. J. Saucedo-Dorantes, M. Delgado-Prieto, J. A. Ortega-Redondo, R. A. Osornio-Rios, R. D. J. Romero-Troncoso, "Multiple-fault detection methodology based on vibration and current analysis applied to bearings in induction motors and gearboxes on the kinematic chain", Shock Vib., vol. 2016, 2016. DOI: 10.1016/j.asej.2017. 06.002.

[9] V. C. M. N. Leite, et al., "Detection of localized bearing faults in induction machines by spectral kurtosis and envelope analysis of stator current", IEEE Trans. Ind. Electron., vol. 62, no. 3, pp. 18551865, 2015. DOI: 10.1109/TIE.2014.2345330.

[10] L. Frosini, C. Harlisca, L. Szabo, "Induction machine bearing fault detection by means of statistical processing of the stray flux measurement", IEEE Trans. Ind. Electron., vol. 62, no. 3, pp. 18461854, 2015. DOI: 10.1109/TIE.2014. 2361115.

[11] E. T. Esfahani, S. Wang, V. Sundararajan, "Multisensor wireless system for eccentricity and bearing fault detection in induction motors", IEEE/ASME Trans. Mechatronics, vol. 19, no. 3, pp. 818826, 2014. DOI: 10.1109/TMECH. 2013.2260865.

[12] A. Shahidi, L. A. Gupta, A. Kovacs, D. Peroulis, "Wireless temperature and vibration sensor for real-time bearing condition monitoring", in IEEE MTT-S International Microwave Symposium Digest, 2013, pp. 11-14.

[13] J. R. Stack, R. G. Harley, T. G. Habetler, “An amplitude Modulation detector for fault diagnosis in rolling element bearings", IEEE Trans. Ind. Electron., vol. 51, no. 5, pp. 1097-1102, 2004. DOI: 10.1109/TIE.2004.834971.

[14] W. Wang, O. A. Jianu, "A smart sensing unit for vibration measurement and monitoring", IEEE/ASME Trans. Mechatronics, vol. 15, no. $1, \quad$ pp. 70-78, 2010. DOI: 10.1109/TMECH.2009.2016956.

[15] M. Hamadache, D. Lee, K. C. Veluvolu, "Rotor speed-based bearing fault diagnosis (RSB-BFD) under variable speed and constant load", IEEE Trans. Ind. Electron., vol. 62, no. 10, pp. 6486-6495, 2015. DOI: 10.1109/TIE. 2015.2416673.

[16] L. Eren, "Bearing fault detection by one-dimensional convolutional neural networks", Math. Probl. Eng., vol. 2017, pp. 1-9, 2017. DOI: $10.1155 / 2017 / 8617315$.

[17] R. Chandralekha, D. Jayanthi, "Diagnosis of faults in three phase induction motor using neuro fuzzy logic", Int. J. Appl. Eng. Res., vol. 11, no. 8, pp. 5735-5740, 2016.

[18] B. Li, M. Y. Chow, Y. Tipsuwan, J. C. Hung, "Neural-network-based motor rolling bearing fault diagnosis", IEEE Trans. Ind. Electron., vol. 47, no. 5, pp. 1060-1069, 2000. DOI: 10.1109/41.873214.

[19] A. S. Raj, N. Murali, "Early classification of bearing faults using morphological operators and fuzzy inference", IEEE Trans. Ind. Electron., vol. 60, no. 2, pp. 567-574, 2013. DOI: 10.1109/TIE.2012.2188259.

[20] J. Zarei, "Induction motors bearing fault detection using pattern recognition techniques", Expert Syst. Appl., vol. 39, no. 1, pp. 68-73, 2012. DOI: 10.1016/ j.eswa.2011.06. 042.

[21] M. E. H. Benbouzid, M. Vieira, C. Theys, "Induction motors' faults detection and localization using stator current advanced signal processing techniques", IEEE Trans. Power Electron., vol. 14, no. 1, pp. 14-22, 1999. DOI: 10.1109/63. 737588.

[22] A. Sapena-Bano et al., "Harmonic order tracking analysis: a novel method for fault diagnosis in induction machines", IEEE Trans. Energy Convers., vol. 30, no. 3, pp. 833-841, 2015. DOI: 10.1109/TEC.2015.2416973.

[23] H. Ohta, N. Sugimoto, "Vibration characteristics of tapered roller bearings", Journal of Sound and Vibration, vol. 190, no. 2, pp. 137147, 1996. DOI: 10.1006/jsvi.1996.0052.

[24] M. F. Yaqub, I. Gondal, J. Kamruzzaman, K. A. Loparo, “Abrasion modeling of multiple-point defect dynamics for machine condition monitoring", IEEE Trans. Reliab., vol. 62, no. 1, pp. 171-182, 2013. DOI: 10.1109/ TR.2013. 2241231.

[25] F. Immovilli, M. Cocconcelli, A. Bellini, R. Rubini, "Detection of generalized-roughness bearing fault by spectral-kurtosis energy of 
vibration or current signals", IEEE Trans. Ind. Electron., vol. 56, no. 11, pp. 4710-4717, 2009. DOI: 10.1109/TIE.2009.2025288.

[26] T. A. Harris, Introduction to Rolling Bearings. John Wiley \& Sons, Inc., 1991.

[27] K. C. D. Kompella, V. G. R. Mannam, S. R. Rayapudi, "DWT based bearing fault detection in induction motor using noise cancellation", $J$ Electr. Syst. Inf. Technol., vol. 3, no. 3, pp. 411-427, 2016. DOI: 10.1016/j.jesit.2016.07.002.

[28] J. A. O. Jordi Cusido, L. Romeral, A. G. E. Javier, A. Rosero, "Fault detection in induction machines using power spectral density in wavelet decomposition", IEEE Trans. Ind. Electron., vol. 55, no. 2, pp. 633-643, 2008. DOI: 10.1109/TIE.2007.911960.

[29] P. F. R. A. W. Galli, G. T. Heydt, "Exploring the power of wavelet analysis", IEEE Comput. Appl. Power, vol. 9, pp. 37-41, 1996. DOI 10.1109/67.539845.

[30] Y. Cai, Y. He, A. Li, J. Zhao, T. Wang, "Application of wavelet to gearbox vibration signals for fault detection", in Proc. 2nd Int. Conf on Advanced Computer Control,Shenyang, 2010, vol. 192, pp. 441444. DOI: 10.1109/ICACC.2010.5486917.

[31] Ii. Bayram, I. W. Selesnick, "Frequency-domain design of overcomplete rational-dilation wavelet transforms", IEEE Trans. Signal Process., vol. 57, no. 8, pp. 2957-2972, 2009. DOI: 10.1109/TSP.2009.2020756.

[32] C. S. Burrus, R. A. Gopinath, H. Guo, Introduction to Wavelets and Wavelet Transforms: A Primer. 1997th ed. Prentice Hall, 1997.

[33] I. Bayram, I. W. Selesnick, "Overcomplete discrete wavelet transforms with rational dilation factors", IEEE Trans. Signal Process., vol. 57, no. 1, pp. 131-145, 2009. DOI:
10.1109/TSP.2008.2007097.

[34] A. Chernenko, E. Ziganshin, "Pulse-doppler uwb radar", in Proc. Int. Conf on Ultrawideband and Ultrashort Impulse Signals, Sevastopol, Ukraine, 2006, pp. 1-3. DOI: 10.1109/UWBUS.2006.307183.

[35] C. Tyren, "Sensor for non-contacting detection via modulation of electromagnetic signal through by measurement entity controlled mechanical resonance", WO 2001073389 A1, 2001.

[36] V. E. Thoren, "Prosthaphaeresis revisited", Hist. Math., vol. 15, no. 1, pp. 32-39, 1988. DOI: $10.1016 / 0315-0860(88) 90047-X$.

[37] S. Faruque, Radio Frequency Modulation Made Easy. Springer International series, 2017, pp. 19-20. DOI: 10.1007/978-3-31941202-3_1.

[38] M. O. Sonnaillon, R. Urteaga, F. J. Bonetto, "Software PLL based on random sampling", IEEE Trans. Instrum. Meas., vol. 59, no. 10, pp. 2621-2629, 2010. DOI: 10.1109/TIM. 2009.2036459.

[39] M. Reddy Barusu, U. Sethurajan, M. Deivasigamani, "A novel lowcost SPLL based electrical faults identification for three-phase squirrel cage induction motor using handheld doppler radar signal analysis", IET Power Electron., 2018, to be published.

[40] M. Lopez-Ramirez et al., "Detection and diagnosis of lubrication and faults in bearing on induction motors through STFT", in Proc. Int. Conf on Electronics, Communications and Computers, Cholula, 2016 , pp. 13-18. DOI: 10.1109/CONIELECOMP.2016.7438545.

[41] J. B. H. Yeolekar, Saket, G. N. Mulay, "Outer race bearing fault identification of induction motor based on stator current signature by wavelet transform", in Proc. 2nd IEEE Int. Conf. On Recent Trends in Electronics Information \& Communication Technology Bengaluru, 2017, pp. 2011-2015. 